\title{
Belphégor
}

\section{Annotazioni belfagoriane (e salgariane)}

\section{Vittorio Frigerio}

\section{(2) OpenEdition}

\section{Journals}

Electronic version

URL: https://journals.openedition.org/belphegor/1399

DOI: 10.4000/belphegor.1399

ISSN: 1499-7185

\section{Publisher}

LPCM

\section{Electronic reference}

Vittorio Frigerio, "Annotazioni belfagoriane (e salgariane)", Belphégor [Online], 16-1 | 2018, Online since 16 July 2018, connection on 19 October 2021. URL: http://journals.openedition.org/belphegor/1399; DOI: https://doi.org/10.4000/belphegor.1399

This text was automatically generated on 19 October 2021.

\section{(c)}

Belphégor est mis à disposition selon les termes de la Licence Creative Commons Attribution - Pas d'Utilisation Commerciale - Pas de Modification 4.0 International. 


\title{
Annotazioni belfagoriane (e salgariane)
}

\author{
Vittorio Frigerio
}

1 Era sotto il titolo "Notules Belphégoréennes" che il nostro rimpianto amico Charles Grivel, cui questo numero è affettuosamente dedicato, soleva proporre le sue riflessioni en vrac su opere e temi più o meno connessi. Riprendiamo oggi questo titolo immaginifico per attirare l'attenzione dei lettori della nostra rivista sulle novità editoriali ancora abbastanza recenti consacrate a Emilio Salgari, il cui numero e la cui qualità restano notevoli e testimoniano della posizione ormai pienamente acquisita dall'autore nel panorama letterario italiano.

2 Per primo, anche cronologicamente, occorre ricordare il volume di Felice Pozzo intitolato Tra Sandokan e Salgari e sottotitolato "Yanez de Gomera, il bohémien dei mari malesi", uscito presso la casa editrice pisana Bibliografia e Informazione nel 2016 (ISBN : 978-88-909833-4-4, 157 p.). Fin dall'introduzione, l'autore esplicita con chiarezza il suo progetto: ridare il posto che merita alla figura dell'avventuriero portoghese, braccio destro del pirata malese, ma, ben lungi dall'essere un semplice comprimario, vero deuteragonista e "alter ego dell'autore, per molti versi" (8). Ci vuole in effetti la notevolissima conoscenza della vita e non solo delle opere del romanziere che sa sempre dimostrare Pozzo, per riuscire a scoprire, o a indovinare, tanti di questi "versi" senza cader mai nel banale appiattimento degli scritti sulle vicende dell'esistenza. L'intuizione che tutti i lettori attenti dei romanzi malesi di Salgari hanno avuto in qualche momento - che "dopo tutto si potrebbe parlare di "ciclo di Yanez"' (139), tanto questo personaggio s'impone con sempre maggior prepotenza all'attenzione dei lettori col proseguire delle avventure - è qui comprovata appieno in un attento quanto piacevole percorso attraverso la saga intera. Pozzo non solo analizza con finezza lo svolgersi intricato delle narrazioni, ma restituisce anche al ciclo la sua mole effettiva, discutendo della scelta mondadoriana di escludere dall'opera i titoli pubblicati postumi, a partire da La riconquista del Mompracem, e mostrando come essa sia stata ingiustificata. Ricostruendo un identikit completo del personaggio attraverso tutte le sue apparizioni, ritraccia anche il "graduale e meditato lavoro sul personaggio" (60) fatto da Salgari, 
riuscendo perfino a suggerire convincenti motivi biografici per spiegare certi cambiamenti nelle abitudini, nel fisico o nell'età del Portoghese da un romanzo all'altro. Ma soprattutto, nel mostrare in modo semplice e convincente le corrispondenze tra la coppia Yanez-Surama e quella Salgari-Ida, egli riesce a rivelare lo spessore autentico del personaggio - viene voglia di dire la sua psicologia, per dar torto a coloro che stimano i romanzi d'avventure incapaci di farne uso. E Pozzo conclude giustamente che è "impossibile trovare nel resto dell'opera salgariana un personaggio cosí a lungo e cosí accuratamente tratteggiato, anche psicologicamente." (151-152). Nutrito al contempo da una rara familiarità con i romanzi e da una conoscenza enciclopedica delle vicende dell'autore, della sua famiglia e del suo ambiente, questo volume si legge con grande interesse e aggiunge una nuova pietra, per nulla trascurabile, all'edificio critico già notevole costruito negli anni da questo attento e appassionato esegeta salgariano.

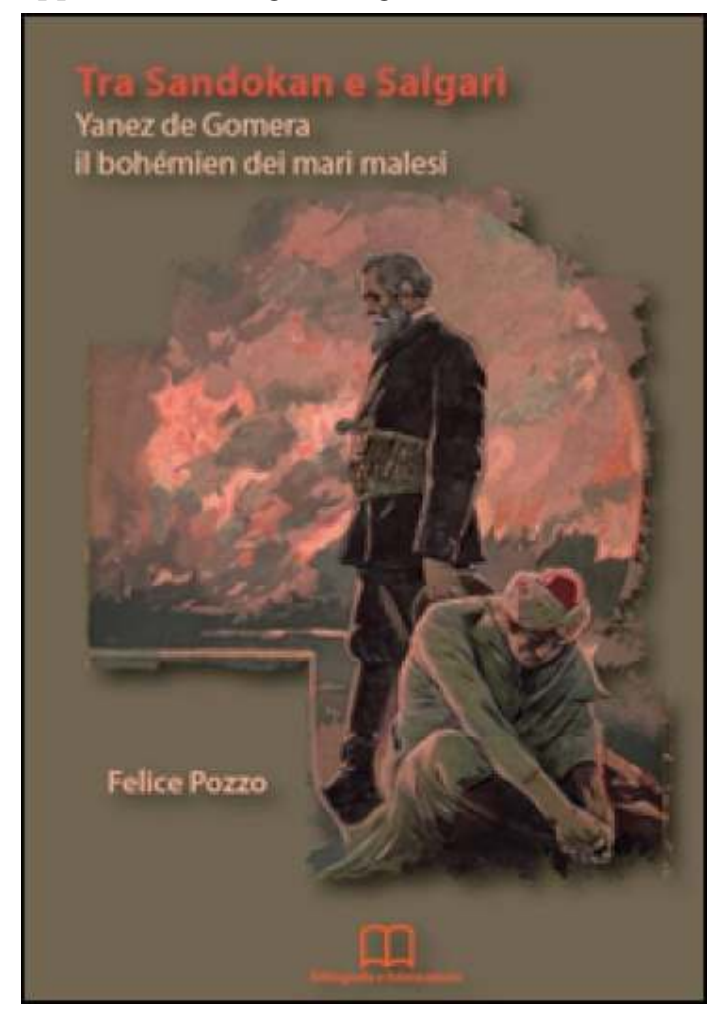

La "vendetta postuma" del romanziere veronese sui suoi detrattori si compie anche attraverso avvenimenti quali l'inclusione di Emilio Salgari nella Storia Mondiale dell'Italia a cura di Andrea Giardina (Bari : Laterza, 2017). In un panorama che va dal 3200 avanti Cristo al 2015, a Salgari tocca rappresentare l'anno 1883, con La Tigre della Malesia (pp.547-550). Paola Irene Galli Mastrodonato vi presenta, con la sua consueta attenzione agli aspetti più "militanti" dell'opera, il ritratto conciso e entusiasta di un autore che si puó leggere "in chiave moderna, libertaria, anticolonialista e multietnica" (547). Un testo breve e denso, pieno di rimandi, che ritrova in Salgari un "elogio del meticciato" molto contemporaneo. 


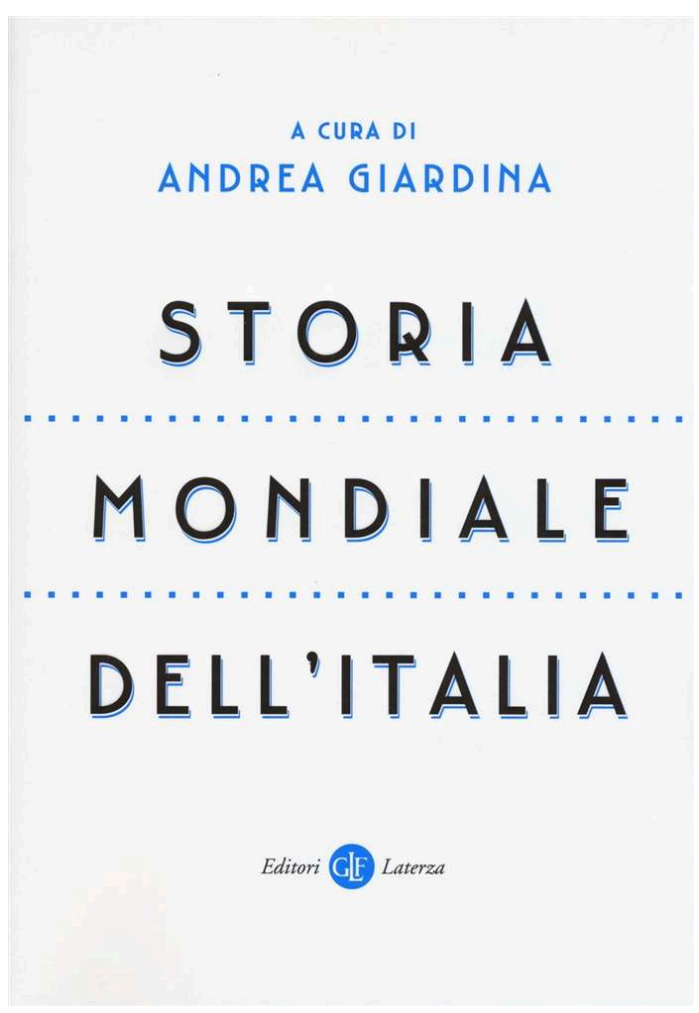

Maurizio Sartor e Claudio Gallo danno alle stampe Lo Stagno dei caimani e altri racconti perduti (Milano: Bompiani, 2018. ISBN 978-88-452-9676-5, 191 p.). Si tratta di nove racconti pubblicati sotto lo pseudonimo Capitano Guido Altieri e ambientati, come dice la quarta di copertina, "negli angoli più remoti del pianeta, dai ghiacci del Polo Nord alle praterie dell'Arkansas ai mari pericolosi della Papuasia." Il tutto incorniciato da un'introduzione di Sartor e di una Postfazione di Giuseppe Bonomi e Claudio Gallo. L'introduzione, spiritosa quanto informativa, ci narra la caccia del suo autore al "racconto salgariano perduto [...] riportato in tutte le bibliografie finora edite : Cap. Guido Altieri, Lo stagno dei caimani, in Psiche. Letture Moderne Illustrate, giugno 1901 [irreperibile]" (6). Ma visto che apparentemente esiste qualche buon genio per gli appassionati (perlomeno quelli persistenti), Sartor riesce non solo a ritrovare il testo misterioso, ma identifica anche uno pseudonimo ancora sconosciuto di Salgari, Giulio Retadi, anagramma di Guido Altieri. È dunque Un principe al Polo Nord, racconto firmato dal misterioso Retadi e pubblicato nello stesso numero di Letture Moderne Illustrate per le Famiglie, pubblicazione più che rarissima, che viene al secondo posto in questa piccola serie di storie dimenticate. La Postfazione ricostruisce con dovizia di particolari la storia della casa editrice Biondo, di Palermo, all'origine tra l'altro della famosa Bibliotechina Aurea Illustrata nella quale i testi salgariani firmati con pseudonimo Altieri essendo il suo preferito - si contano a decine, e rivela l'importanza di questo alter ego, che chiamava Salgari "zio" nella corrispondenza con gli editori, e che costui chiamava scherzosamente "mio nipote". In quanto ai racconti stessi, tutti di gradevole lettura e nei quali i temi classici salgariani si identificano facilmente, ognuno avrà il suo preferito. Si può segnalare, per il suo interesse e per la rarità del soggetto, l'ultimo della serie, Il mio terribile segreto, a firma Enrico Bertolini, che marca un'incursione di Salgari in un mondo che ha frequentato ben poco : quello del fantastico. 


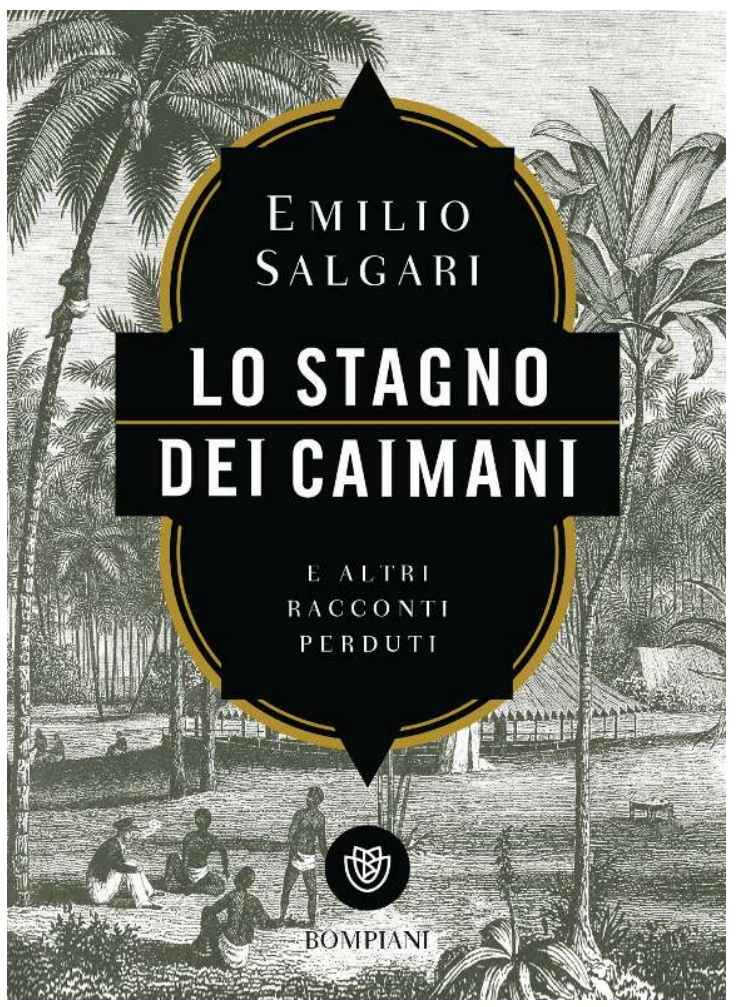

AUTHOR

VITTORIO FRIGERIO

Dalhousie University 